PAPER

\title{
Neurosyphilis in the modern era
}

\section{Timmermans, J Carr}

J Neurol Neurosurg Psychiatry 2004;75:1727-1730. doi: 10.1136/jnnp.2004.031922

See end of article for authors' affiliations .....................

Correspondence to:

Dr J Carr, Neurology Unit, Tygerberg Hospital, Tygerberg 7505, South Africa; jcarr@sun.ac.za

Received

26 November 2003

Revised 25 February 2004

Accepted 9 March 2004

\begin{abstract}
Objective: To review the nature of the presentation of neurosyphilis, the value of diagnostic tests, and the classification of the disease.

Methods: A retrospective review was carried out of the records of patients who had been identified as possible cases of neurosyphilis by a positive FTA-abs test in the CSF. The review extended over 10 years at a single hospital which served a population of mixed ancestry in a defined catchment area in the Western Cape province of South Africa. Patients were placed in predefined diagnostic categories, and clinical, radiological, and laboratory features were assessed.

Results: 161 patients met diagnostic criteria for neurosyphilis: 82 presented with combinations of delirium and dementia and other neuropsychiatric conditions, and the remainder had typical presentations such as stroke (24), spinal cord disease (15), and seizures (14). The average age of presentation ranged from 35.9 to 42.6 years in the different categories of neurosyphilis. Of those followed up, $77 \%$ had residual deficits from their initial illness. Cerebrospinal fluid (CSF) VDRL was positive in $73 \%$ of cases.

Conclusions: The diagnosis of neurosyphilis can be made with reasonable certainty if there is an appropriate neuropsychiatric syndrome associated with a positive CSF VDRL. If the VDRL is negative, a positive FTA-abs in an appropriate clinical setting, associated with raised CSF cell count, protein, or lgG index, is a useful method of identifying neurosyphilis. Tabes dorsalis has become uncommon, but this is likely to be the only manifestation of neurosyphilis that has been altered during the antibiotic era.
\end{abstract}

$\mathrm{N}$ eurosyphilis appears to be common in South Africa, in contrast to the developed world, where it is a rare disease. ${ }^{12}$ Accurate diagnosis remains problematic, as the signs are not pathognomonic and the sensitivity and specificity of supportive laboratory tests are unclear. Uncertainty about the diagnosis is compounded by reports that the manifestations of neurosyphilis have altered with the widespread use of antibiotics. ${ }^{34}$ Similarly, it has been reported that neurosyphilis has a more fulminant course and is resistant to treatment when associated with HIV infection. ${ }^{5-7}$ In the last decade there has been a 50 -fold increase in syphilis in eastern Europe, ${ }^{8}$ and epidemics continue to occur with a seven to 10 year cycle in the USA.

In this paper we review the diagnostic features and laboratory findings in 161 patients with neurosyphilis, to our knowledge the largest such series described since the onset of the antibiotic era. The purpose of our review was to examine the ways in which neurosyphilis may present and the ages of presentation, and to reassess the traditional classification.

\section{METHODS}

The FTA-abs test (fluorescent treponema antibody testabsorption) in the cerebrospinal fluid (CSF) is a highly sensitive marker for the presence of neurosyphilis. ${ }^{9-11}$ We therefore obtained records on all patients with positive FTAabs results in the CSF from 1990 to 1999 at the Tygerberg Hospital, Cape Town, South Africa. These were extracted from a computerised hospital based data system, noting that the hospital has a geographically defined catchment area and provides both paediatric and psychiatric services. Peripheral clinics-which serve both a large urban and a mainly farm based rural community-are the predominant source of referrals. The referral population is characterised by a high rate of illiteracy and alcoholism, poor access to health care, and some of the highest rates of pulmonary tuberculosis and, more recently, HIV infection in the world. The hospital has traditionally served a population of mixed ancestry, whose origins include indigenous peoples of southern Africa, predominantly the Khoi-San and Xhosa speaking black Africans, immigrants of European descent, and slaves from former Dutch colonies in Malaysia.

Patients with congenital syphilis were excluded from the study. Data were extracted on age, sex, clinical presentation of symptoms, duration of symptoms, treatment, outcome, recurrence, blood and CSF fluid serology, CSF picture at presentation and follow up, special investigations, and HIV status. Special investigations included computed tomography (CT) and magnetic resonance imaging (MRI).

Clinical and laboratory features were entered into templates for six diagnostic categories, which were derived from our previous experience with neurosyphilis, review of published reports, and a pilot study:

Category $1-$ Neuropsychiatric disorders: including psychosis, delirium, and dementia.

Category 2-Cerebrovascular accident (CVA): acute, focal neurological deficit, compatible with a CVA or radiological evidence of stroke.

Category 3-Ocular: presentation with uveitis, visual loss, or optic nerve dysfunction.

Category 4-Myelopathy: acute, subacute, or chronic dysfunction of the spinal cord, including tabes dorsalis.

Category 5-Seizure: presentation with partial seizures, with or without secondary generalisation, or myoclonus.

Category 6-Brain stem/cranial nerves: signs restricted to the brain stem and cranial nerves.

In addition to the primary clinical diagnostic group, overlap with other clinical groups was also recorded.

Abbreviations: FTA(-abs), fluorescent treponema antibody test (-absorption); GPI, general paresis of the insane; HIV, human immunodeficiency virus; HTLV-1, human T cell lymphotropic virus type I; PML, polymorphonuclear leucocytes; RPR, rapid plasma reagin; TBM, tuberculous meningitis; VDRL, Venereal Disease Research Laboratory 
Patients who had appropriate clinical features and met the following inclusion criteria were allocated to the category of neurosyphilis. ${ }^{12} 13$

- Positive cerebrospinal fluid VDRL (Venereal Disease Research Laboratory) or

- Positive cerebrospinal fluid FTA-abs, with either

- Abnormal CSF cell count (polymorphonuclear leucocytes (PML) and/or lymphocytes $>5 / \mathrm{ml}$ ), or

- CSF protein $>0.45 \mathrm{~g} / \mathrm{l}$, or

- IgG index $>0.6$.

\section{RESULTS}

There were 324 cases with positive FTA-abs serology in the CSF between 1990 and 1999, and 161 met diagnostic criteria for inclusion in this study as cases of neurosyphilis. Of the remaining 163 cases 12 had inadequate information to determine their status. The rest were excluded for the following reasons.

In 95 patients the CSF protein, cell count, and IgG index were normal, although five of these were noted to have weakly positive VDRL results in the CSF (diagnoses were traumatic lumbar puncture, bronchial carcinoma with metastases to skull base, axonal polyneuropathy, renal failure, and dementia); 20 patients had increased cells and protein in the CSF, with diagnoses of tuberculous meningitis (TBM) (8), cryptococcal meningitis (1), bacterial meningitis (2), S aureus septicaemia (1), Guillain-Barré syndrome associated with HIV (1), human T cell lymphotropic virus type 1 (1), status epilepticus (1), stroke (2), and traumatic lumbar puncture (3).

Nineteen patients had raised protein only in CSF, with diagnoses including Guillain-Barré syndrome (2), polyradiculopathy (1), HIV related polyneuropathy (1), chronic inflammatory demyelinating polyradiculopathy (1), cervical spondylosis (2), hypertensive encephalopathy (1), status epilepticus (1), diabetes mellitus (1), transverse myelitis (1), dementia (1), spinal cord meningioma (1), stroke (4), and traumatic lumbar puncture (2).

Six patients had an increased cell count only, related to stroke (2), seizure (3) and traumatic lumbar puncture (1).

Five patients had a raised IgG index only, with diagnoses including mental retardation, suicide attempt, alcoholic cerebellar disorder, seizures, and possible tabes dorsalis (in this case the CSF was entirely normal except for an IgG index of 0.69 ).

Six patients had raised VDRL titres in their CSF, associated with raised protein and cell count, but two had culture proven bacterial meningitis, one had a subarachnoid haemorrhage related to an aneurysm, and three had hydrocephalus with a markedly abnormal CSF and responded to antituberculosis treatment.

Among the patients whom we considered to have an established diagnosis of neurosyphilis, six were found to be HIV positive, while 96 tested negative for HIV. Regarding the origin of the patients with neurosyphilis, 140 were of mixed ancestry and the remaining 21 were of black African descent.

The neurosyphilis patients were divided into diagnostic groups (table 1): 82 cases had a neuropsychiatric presentation and the remaining groups ranged from CVA (24) to brain stem/cranial nerve involvement (8). In the spinal cord group, one patient had definite tabes dorsalis, and one had a possible diagnosis of tabes.

Overlap between different diagnostic groups was largely restricted to those presenting with seizure, stroke, or neuropsychiatric features. Thus $11 \%$ of the neuropsychiatric

\begin{tabular}{|c|c|c|}
\hline & n (\%) & Age (years)* \\
\hline Neuropsychiatric & $82(50.9)$ & $41(10.35)$ \\
\hline CVA & 24 (14.9) & $36(9.39)$ \\
\hline Ocular & 18 (11.9) & $37.2(10.18)$ \\
\hline Myelopathy & $15(9.3)$ & $35.9(10.61)$ \\
\hline Seizure & $14(8.7)$ & $42.6(12.11)$ \\
\hline Brain stem/cranial nerves & $8(5.0)$ & $40.87(9.45)$ \\
\hline
\end{tabular}

group also had seizures, and $43 \%$ of the seizure group and $29 \%$ of the CVA group also had a delirium.

The age of onset of the various diagnostic categories showed substantial overlap (fig 1), with an average age of 39 years (range 17 to 67).

The average duration of treatment was 14.9 days in hospital, the commonest treatment being 20 million units of penicillin G daily. Evidence of recurrence of neurosyphilis, based on clinical deterioration, a rising CSF VDRL titre, or worsening of markers of disease activity in CSF, was found in 20 patients $(12 \%)$.

The outcome was known in 92 cases. Twenty one patients recovered completely, 40 suffered from residual cognitive loss, 10 had psychiatric disorders, seven had residual seizures, seven had residual hemiparesis, one suffered from tabes dorsalis, and six died.

\section{Laboratory diagnosis}

In the neurosyphilis group (table 2), 108 VDRLs were recorded in serum, of which 106 were positive and 89 showed significant titres of $>1: 8$. The serum FTA was positive in all cases in which the test was done. In the 149 cases in whom a CSF-VDRL was done, it was positive in 109 cases but in only 19 was the titre greater than 1:8. Of note, $68 \%$ of cases had an IgG index of greater than 1 .

The majority of patients with a neuropsychiatric presentation had generalised cerebral atrophy with ventricular dilatation on CT and MRI (34 patients, of 41 who were scanned) (fig 2). Of 18 patients with CVA and imaging, seven had a middle cerebral artery territory stroke and eight had lacunar strokes (fig 3). MRI was particularly useful in demonstrating meningeal enhancement, which tended to be patchy and thin.

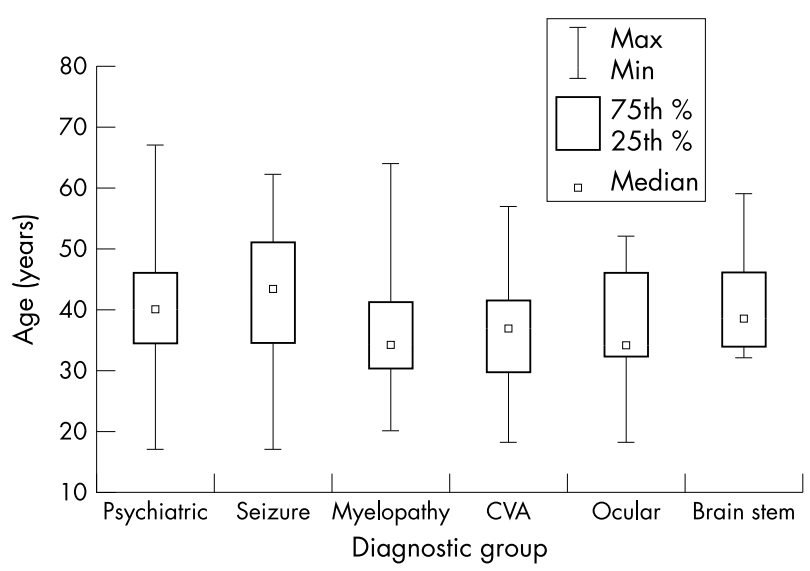

Figure 1 Box and whisker plot of the ages of presentation of the diagnostic categories of neurosyphilis. 
Table 2 Markers of activity and serological status in the neurosyphilis group

\begin{tabular}{lllll}
\hline & $\mathbf{n}$ & Positive $(\mathbf{n}(\%))$ & $\begin{array}{l}\text { Significant value } \\
(\mathbf{n}(\%))\end{array}$ & Mean (range) \\
\hline Serum VDRL & 108 & $106(98)$ & $89(84)$ & $126(2$ to 512$)$ \\
Serum RPR & 48 & $46(96)$ & $40(87)$ & $149(1$ to 1024) \\
Serum FTA-abs & 72 & $72(100)$ & - & - \\
CSF VDRL & 149 & $109(73)$ & $19(13)$ & $8.6(1$ to 64$)$ \\
CSF protein & 150 & $123(82)$ & $79(53)$ & $0.98(0.12$ to 10.0$)$ \\
CSF cell count & 156 & $118(76)$ & $76(49)$ & $60(0$ to 1022) \\
CSF lgG index & 85 & $77(91)$ & $58(68)$ & $2.41(0.21$ to 13.97) \\
\hline Significant value: VDRL titre $>1: 8$, protein $>0.8 \mathrm{~g} / \mathrm{l})$ cell count $>25 / \mathrm{ml}$ lgG index $>1$. \\
FTA-abs, fluorescent treponema antibody test-absorption; RPR, rapid plasma reagin; VDRL, venereal disease \\
research laboratory
\end{tabular}

\section{DISCUSSION}

Neurosyphilis has become a rare disorder in the developed world. This, coupled with the use of ill defined terminology and uncertainty concerning the diagnostic value of available tests, can result in confusion for the clinician. On the basis of this study, we believe that we can clarify certain issues.

\section{What is the best way to characterise and classify neurosyphilis?}

We classified patients into groups depending on their mode of presentation, which reflected their predominant clinical syndromes. The advantage of a clinically based syndromic classification is that it makes intuitive sense, even though overlap in clinical symptoms may be present. This scheme of classification compares to the common practice of a pathological diagnosis being assigned to a clinical presentation, ${ }^{14}$ which usually corresponds to educated guesswork as pathological samples are not normally obtained in the course of the diagnostic work up for neurosyphilis. The traditional terminology used for neurosyphilis lacks precise definition, and pathologically many cases may have meningeal, vascular,

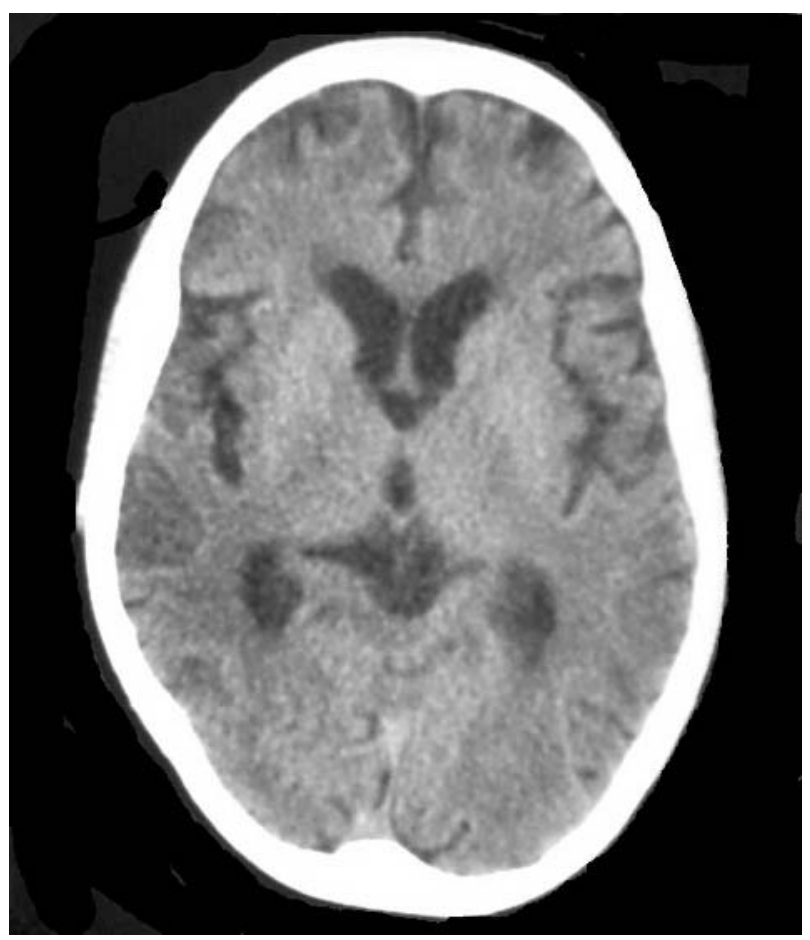

Figure 2 Computed tomography of the brain of an 18 year old patient who presented with dementia. Serum VDRL (Venereal Disease Research Laboratory) was 1:256, cerebrospinal fluid (CSF) VDRL was positive, CSF protein was $0.89 \mathrm{~g} / \mathrm{l}$, and the CSF $\mathrm{lgG}$ index was 6.39 . and parenchymal involvement. Although MRI may contribute to a more precise anatomical diagnosis, neurosyphilis is probably still best classified by the nature of the predominant clinical deficit, as is the case with other forms of chronic meningitis. ${ }^{15}$ This method of classification would give rise to terms such as "neurosyphilis with seizures, ....with stroke, ....with cranial nerve involvement, ....with spinal involvement". General paresis of the insane (GPI) is now an outmoded term, and a preferable description might be "syphilitic encephalitis."

\section{What is the latency of the various manifestations of neurosyphilis?}

The classification of neurosyphilis has often been interwoven with the concept that the various manifestations of the condition are time dependent. For example, with regard to the syndromes of meningeal and meningovascular syphilis, GPI, and tabes dorsalis, ${ }^{16}$ reviews of neurosyphilis have suggested that the latency from initial infection to the

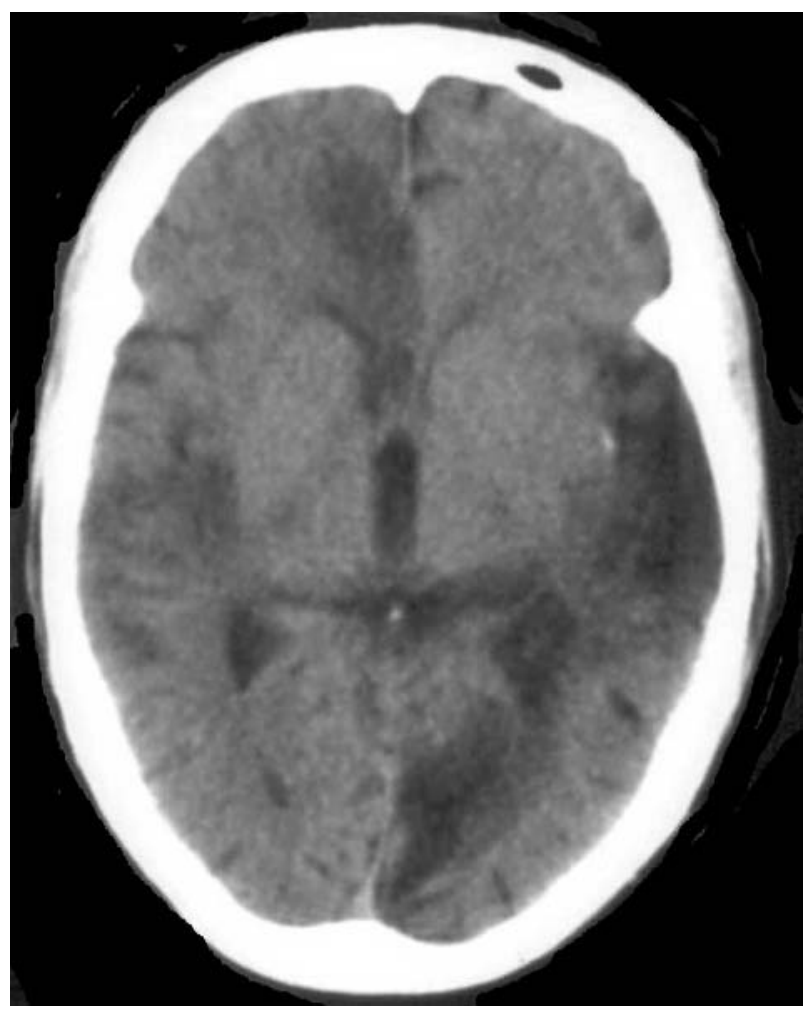

Figure 3 Computed tomography of the brain of a 43 year old HIV positive patient who presented with hemiparesis. Serum RPR (rapid plasma reagin) was 1:256, cerebrospinal fluid (CSF) VDRL was positive, and CSF protein was $1.57 \mathrm{~g} / \mathrm{l}$, with 14 lymphocytes $/ \mathrm{ml}$. 
development of these four syndromes is, respectively, a few months, five years, 10 years, and 15 to 20 years. ${ }^{17}$ In the current study, no appreciable difference was observed in the ages of presentation, regardless of the syndrome (fig 1). The average age of presentation for the two purported extremes of neurosyphilis-namely meningeal (brain stem) and neuropsychiatric (the latter corresponding to GPI)—were 40.9 and 41 years, respectively.

It is likely that age correlates with duration of illness, and it may be that age of onset is a more accurate reflection of disease duration than the estimate of duration derived from patient recall of their evidence of primary or secondary infection.

\section{How is neurosyphilis diagnosed?}

The diagnosis of neurosyphilis, or more often the definite exclusion of neurosyphilis as a clinical possibility, remains a difficult problem. It is likely, and widely accepted, that a positive VDRL in the CSF is sufficient to diagnose neurosyphilis, although false positive results caused by contamination from serum are possible. ${ }^{18}$ What if the CSF VDRL is negative? In this study, $27 \%$ of patients with typical symptoms of neurosyphilis had a combination of a negative VDRL and a positive FTA in the CSF. However, a high proportion of cases with a positive FTA in the CSF did not have neurosyphilis. There is no gold standard for the diagnosis of neurosyphilis, and clinical uncertainty is likely to persist, particularly if the diagnosis rests on a test which is relatively poorly specific, as the CSF FTA appears to be. However, the FTA may be the only serological evidence of the occurrence of neurosyphilis, and carries the advantage of being highly sensitive. The utility of a test is dependent on the background prevalence of the condition, and in populations where neurosyphilis is rare, the number of false positive cases is likely to be greater. The primary value of the FTA test in CSF may be to exclude the possibility of neurosyphilis if the test result is negative.

\section{Has the presentation of neurosyphilis changed in the antibiotic era?}

In the pre-penicillin era, tabes dorsalis was the commonest manifestation of neurosyphilis. ${ }^{19} 20$ Tabes appears to have been a chronic, indolent, and in some cases, rather mild illness, and might therefore-of all the manifestations of neurosyphilis-be the most susceptible to antibiotics. Additionally, apart from errors of diagnosis in what is sometimes a rather ill defined syndrome, ascertainment bias may have led to overestimation of the frequency of tabes. ${ }^{19}$ Since the onset of the antibiotic era, the spectrum of neurosyphilis is reported to have changed, as suggested by a study by Hooshmand et al in which 241 new cases of neurosyphilis were reported. ${ }^{3}$ However, the majority of these were asymptomatic, and the remainder had atypical syndromes, ${ }^{3}$ with only $49 \%$ having a reactive non-treponemal serum test for syphilis. A study from South Africa reported that the majority of patients with neurosyphilis presented with subtle clinical signs and with weakly positive or even negative serology, ${ }^{4}$ and a subsequent editorial in the $B M J$ in $1978^{21}$ suggested that atypical neurosyphilis may be common. In contradistinction, a study from the United Kingdom in
1979 reported 17 cases of neurosyphilis and found that the presentations were not atypical..$^{22}$ Wolters compared pre- and post-antibiotic era patients, and apart from a decline in tabes dorsalis, no other significant differences were seen. ${ }^{23}$ The present study indicates that the classical presentations of neurosyphilis have not altered, although tabes dorsalis has become rare. Our patients had clinical syndromes that were identical to those described in the pre-antibiotic era-that is, neuropsychiatric presentations, stroke, cranial nerve and brain stem dysfunction, seizures with or without encephalopathy, and spinal cord disease, both acute and indolent. However, as opposed to the developed world, the population studied in this report has significantly poorer access to health care, and is less likely to have received similar quantities of antibiotics.

\section{Authors' affiliations}

M Timmermans, J Carr, Neurology Unit, University of Stellenbosch, South Africa

Competing interests: none declared

\section{REFERENCES}

1 Pham-Kanter GB, Steinberg MH, Ballard RC. Sexually transmitted diseases in South Africa. Genitourin Med 1996;72:160-71.

2 Routine tests for syphilis on cerebrospinal fluid [editorial]. Lancet 1977;ii:595.

3 Hooshmand H, Escobar MR, Kopf SW. Neurosyphilis. A study of 241 patients. JAMA 1972;219:726-9.

4 Joyce CN, Molteno AC. Modified neurosyphilis in the Cape Peninsula. S Afr Med J 1978;53:10-14.

5 Berry CD, Hooton TM, Collier AC, et al. Neurologic relapse after benzathine penicillin therapy for secondary syphilis in a patient with HIV infection. N Engl J Med 1987;316:1587-9.

6 Johns DR, Tierney M, Felsenstein D. Alteration in the natural history of neurosyphilis by concurrent infection with the human immunodeficiency virus. N Engl J Med 1987:316:1569-72.

7 Katz DA, Berger JR. Neurosyphilis in acquired immunodeficiency syndrome [see comments]. Arch Neurol 1989;46:895-8.

8 Tichonova L, Borisenko K, Ward H, et al. Epidemics of syphilis in the Russian Federation: trends, origins, and priorities for control. Lancet 1997;350:210-13.

9 Deacon WE, Lucas JB, Price EV. Fluorescent treponemal antibody-absorption (FTA-ABS) test for syphilis. JAMA 1966;198:624-8.

10 Escobar MR, Dalton HP, Allison MJ. Fluorescent antibody tests for syphilis using cerebrospinal fluid: clinical correlation in 150 cases. Am J Clin Pathol 1970;53:886-90

11 Leclerc G, Giroux M, Birry A, et al. Study of fluorescent treponemal antibody test on cerebrospinal fluid using monospecific anti-immunoglobulin conjugates $\lg G, \lg M$, and $\lg A$. Br J Vener Dis 1978;54:303-8.

12 Russouw HG, Roberts MC, Emsley RA, et al. The usefulness of cerebrospinal fluid tests for neurosyphilis. S Afr Med J 1994;84:682-4

13 Roberts MC, Emsley RA, Jordaan GP. Screening for syphilis and neurosyphilis in acute psychiatric admissions. S Afr Med J 1992;82:16-18.

14 Burke JM, Schaberg DR. Neurosyphilis in the antibiotic era. Neurology 1985;35:1368-71.

15 Reik L. Lyme disease. In: Scheld WM, Whitley RJ, Durack DT, eds. Infections of the central nervous system. New York: Lippincott-Raven, 1997:685-718.

16 Simon RP. Neurosyphilis. Arch Neurol 1985;42:606-13.

17 Hook EW, Marra CM. Acquired syphilis in adults. N Engl J Med 1992:326:1060-9.

18 Hart G. Syphilis tests in diagnostic and therapeutic decision making. Ann Intern Med 1986; 104:368-76.

19 Merritt HH, Adams RD, Solomon HC. Neurosyphilis. New York: Oxford University Press, 1946.

20 Wilson SAK. Neurology. London: Butterworth, 1940

21 Modified neurosyphilis [editorial]. BMJ 1978;2:647-8.

22 Luxon L, Lees AJ, Greenwood RJ. Neurosyphilis today. Lancet 1979;i:90-3.

23 Wolters EC. Neurosyphilis: a changing diagnostic problem? Eur Neurol 1987;26:23-8. 